\title{
BMJ Open Rationale and design of a prospective, observational, multicentre study on the safety and efficacy of apixaban for the prevention of thromboembolism in adults with congenital heart disease and atrial arrhythmias: the PROTECT-AR study
}

\author{
Anastasios Kartas (1) , ${ }^{1}$ loannis Doundoulakis, ${ }^{1}$ Despoina Ntiloudi, ${ }^{1}$ \\ Athanasios Koutsakis, ${ }^{1}$ Diamantis Kosmidis, ${ }^{1}$ Georgios Rampidis, ${ }^{1}$ \\ Sotiria Apostolopoulou, ${ }^{2}$ Alexandra Frogoudaki, ${ }^{3}$ Afrodite Tzifa, ${ }^{4,5}$ \\ Dimosthenis Avramidis, ${ }^{4}$ Olga Ntzoyvara, ${ }^{2}$ Sotiria Liori, ${ }^{3}$ Tereza Mousiama, ${ }^{4}$ \\ Sophia Anastasia Mouratoglou, ${ }^{1}$ Haralambos Karvounis, ${ }^{1}$ George Giannakoulas ${ }^{1}$
}

To cite: Kartas A,

Doundoulakis I, Ntiloudi D, et al. Rationale and design of a prospective, observational, multicentre study on the safety and efficacy of apixaban for the prevention of thromboembolism in adults with congenital heart disease and atrial arrhythmias: the PROTECT-AR

study. BMJ Open

2020;10:e038012. doi:10.1136/ bmjopen-2020-038012

- Prepublication history for this paper is available online. To view these files, please visit the journal online (http://dx.doi. org/10.1136/bmjopen-2020038012).

Received 24 February 2020

Revised 02 August 2020 Accepted 03 August 2020

Check for updates

(c) Author(s) (or their employer(s)) 2020. Re-use permitted under CC BY-NC. No commercial re-use. See rights and permissions. Published by BMJ.

For numbered affiliations see end of article.

Correspondence to Dr George Giannakoulas; ggiannakoulas@auth.gr

\section{ABSTRACT}

Introduction The risk for stroke in adults with congenital heart disease (ACHD) is increased, especially in the setting of commonly ensuing atrial arrhythmias (AA), namely atrial fibrillation, atrial flutter or intra-atrial re-entrant tachycardia. Data are limited regarding treatment with non-vitamin $\mathrm{K}$ oral anticoagulants in long-term studies involving patients with ACHD and AA.

Methods and analysis PReventiOn of

ThromboEmbolism in Adults with Congenital HearT disease and Atrial aRrhythmias is a prospective, multicenter, single-arm, non-interventional cohort study designed to investigate the safety and efficacy of apixaban for the prevention of thromboembolism in $A C H D$ with $A A$ in a 'real-world' setting. Eligible patients will be evaluated by the means of available registries and clinical counter. The study aims to accumulate approximately 500 patient-years of exposure to apixaban as part of routine care. Enrolment will take place at four ACHD centres in Greece. The first patient was enrolled in July 2019. The primary efficacy endpoint is a composite of stroke, systemic or pulmonary embolism and intracardiac thrombosis. The primary safety endpoint is major bleeding, according to the International Society on Thrombosis and Haemostasis bleeding criteria.

Ethics and dissemination The study protocol has been approved by the institutional review board/ independent ethics committee at each site prior to study commencement. All patients will provide written informed consent. Results will be disseminated at scientific meetings and published in peer-reviewed journals.

Trial registration number NCT03854149; Pre-results.

\section{Strengths and limitations of this study}

- The multicentre, prospective design will capture high-quality and detailed 'real-world' data on apixaban use in adults with congenital heart disease (ACHD) and atrial arrhythmias.

- The study will exploit available ACHD registries to facilitate patient enrolment

- Five-hundred patient-years will be the largest exposure to a single anticoagulant to be reported in an ACHD study.

- Formal non-inferiority of apixaban compared with warfarin cannot be established by an explanatory study with small sample size and lack of a comparator arm.

- Sample representativeness may be affected by patient-related or physician-related selection bias.

\section{INTRODUCTION}

Adults with congenital heart disease (ACHD) carry a more than 10-fold increased risk for stroke compared with the general population. ${ }^{1}$ This excess risk is partially conveyed by atrial arrhythmias (AAs), namely atrial fibrillation, atrial flutter or intra-atrial re-entrant tachycardia, which are three times more prevalent in ACHD. ${ }^{2}$ Notably, AAs occur in over $10 \%$ of patients within 10 years from the Fontan procedure. ${ }^{3}$ Hence, contemporary guidelines suggest oral anticoagulation (OAC) in most patients with ACHD with AA, based on disease complexity and presence of conventional stroke risk factors. ${ }^{4}$ 
The risk of thrombotic, but also bleeding complications can be even higher for cyanotic entities of the heterogeneous congenital heart disease (CHD) spectrum, mandating safe and effective OAC treatment. ${ }^{5}$ The adoption of non-vitamin K OACs (NOACs) into widespread clinical practice is expected to revolutionise the care of patients with AA-complicated CHD. Apixaban was the third NOAC that was licensed for non-valvular atrial fibrillation, which demonstrated superiority in both the efficacy endpoint of stroke or systemic embolism and in the safety endpoint of major bleeding, with a concomitant $11 \%$ reduction in all-cause mortality versus warfarin. ${ }^{6}$ The landmark trial of apixaban did not exclude patients with ACHD, although no ACHD-specific subgroup analyses have been reported so far. Yet even after a decade on the market, most data generated by studies on ACHD patients with AA focused on NOACs in general, rather than apixaban per se. ${ }^{7-10}$

To address this gap in knowledge, we designed the PReventiOn of ThromboEmbolism in Adults with Congenital HearT disease and Atrial aRrhythmias (PROTECT-AR) trial. The trial aims to evaluate the safety and efficacy of apixaban use for thromboembolism prevention, in routine, 'real-world' practice regarding ACHD patients with AA.

\section{METHODS AND ANALYSIS \\ Study design}

PROTECT-AR is an investigator-initiated, prospective, multicenter, single-arm, non-interventional cohort study involving ACHD patients with AA who are treated with apixaban as part of routine care. The planned enrolment is for 250 patients across four study sites, which are major ACHD centres (American Hellenic Educational Progressive Association [AHEPA], Onassis, Attikon and Mitera Hospital) located in the two largest cities of Greece. The first patient was enrolled in PROTECT-AR on 5 July 2019. As of February 2020, 91 patients have been enrolled. Completion of enrolment is anticipated in the first quarter of 2023. Results of the study are expected until the end of 2023.

The steering committee, consisting of academic authors with expertise in ACHD and in anticoagulation, will be responsible for study oversight, maintenance of the study database, data validation and analyses. The committee is supported by an independent statistician and is responsible for ensuring timely publication of the results. The development of the manuscript was led by the first author, in collaboration with all the authors. No one who is not an author interfered with writing the manuscript. All the authors made the decision to submit the manuscript for publication.

\section{PATIENTS}

To participate in PROTECT-AR, patients must be adults ( $\geq 18$ years old) with CHD and a documented history of atrial fibrillation, atrial flutter or intra-atrial re-entrant tachycardia, who are routinely treated with apixaban (ie, new or ongoing users) for stroke prevention at any healthcare level in Greece (ie, not restricted to the study's sites). Patients will be excluded if they have moderate-tosevere mitral stenosis or mechanical valves, or if they are unwilling or unable to complete follow-up procedures.

\section{ENDPOINTS}

\section{Primary endpoints}

The primary efficacy endpoint is the incidence of composite events, including stroke (ischaemic, haemorrhagic or undetermined), systemic or pulmonary embolism and intracardiac thrombosis. The primary safety endpoint is the incidence of major bleeding.

\section{Secondary endpoints}

The predefined secondary efficacy endpoints include each component of the primary efficacy endpoint, as well as transient ischaemic attack, myocardial infarction, death from cardiovascular causes and death from any cause. The predefined secondary safety endpoint includes the composite of major and clinically relevant nonmajor bleeding.

\section{Endpoint adjudication}

All efficacy and safety endpoints will be adjudicated on the basis of prespecified definitions by a clinical endpoint committee, whose members will have access to all patient records (eg, hospital discharge summary, outpatient clinic letter, brain imaging report or other relevant information), but will not interfere with the patients' care. The study's endpoints, defined in accordance with recent American College of Cardiology and International Society on Thrombosis and Hemostasis Guidelines ${ }^{11-13}$ and used in several key thromboembolism trials, ${ }^{14-16}$ are provided in table 1 .

\section{EXPLORATORY ENDPOINTS}

The clinical thromboembolic or bleeding events that occurred prior to enrolment will be assessed retrospectively. During the follow-up, all adverse events occurring in patients on apixaban treatment, apart from the study's primary and secondary endpoints, will be recorded and analysed. Furthermore, healthcare resource use and details on interventions, such as management of thromboembolic and bleeding events, will be assessed. Apixaban persistence, reasons for any switch from, interruption or cessation of apixaban therapy and any follow-on therapy will be recorded. Adherence to apixaban treatment will be assessed by reviewing electronic pharmacy prescription data in order to track the number of apixaban prescriptions that individual patients claimed. In addition, changes in the quality-of-life of patients who, newly initiated or were switched to apixaban at enrolment, will be 
Table 1 Clinical events definitions

Stroke

Defined as a new-onset neurological dysfunction caused by central nervous system injury as a result of haemorrhage or infarction, of at least 24 hours duration (or if symptoms lasted <24 hours a clear matching lesion on CT or MRI), not due to another identifiable nonvascular cause (ie, brain tumour, trauma).

All available data will be considered to support the diagnosis and subclassification of the type of stroke. Strokes will be subclassified as 'ischaemic'or 'primary haemorrhagic' based on imaging data, if available, or 'undetermined cause' if imaging data are missing, according to the definitions below.

Ischaemic stroke

- Ischaemic stroke with no haemorrhage: stroke without intracerebral blood on brain imaging

- Ischaemic stroke with haemorrhagic conversion: presence of blood felt to represent haemorrhagic conversion and not a primary haemorrhage

Primary haemorrhagic stroke

- A stroke with documentation on imaging (eg, CT scan or MRI) of intracerebral, subdural or subarachnoid haemorrhage. Evidence of haemorrhagic stroke from other sources (lumbar puncture, neurosurgery or autopsy) can also confirm the diagnosis.

Undetermined Stroke

- A stroke as a result of haemorrhage or infarction but with insufficient information classify as either ischaemic or haemorrhagic.

Systemic embolism $\quad$ history consistent with an acute cessation of blood flow to a peripheral artery (or arteries) or evidence of embolism from
other sources (eg, surgical specimens, angiography, vascular imaging), localised to one of the following:
Lower or upper limb
Intraocular
Intra-abdominal viscera
Other (to be specified)

Pulmonary embolism

Symptoms of PE with one of the following findings:

- A new intraluminal filling defect in (sub)segmental or more-proximal branches on CTPA, or in vessels more than $2.5 \mathrm{~mm}$ in diameter on the pulmonary angiogram.

- A new perfusion defect of at least $75 \%$ of a segment with a local normal ventilation result (high probability) on VQ scan.

- Inconclusive diagnosis of PE based on CTPA, pulmonary angiography, or VQ scan, but with demonstration of a new or recurrent deep-vein thrombosis in the lower extremities by compression ultrasound or venography.

\begin{tabular}{|c|c|}
\hline us & $\begin{array}{l}\text { entified by echocardiography or cardiac MRI as a discrete echodense mass with well-defined borders that are distinct from } \\
\text { e endocardium and seen throughout systole and diastole, in any of the four cardiac chambers (including atrial appendages) }\end{array}$ \\
\hline $\begin{array}{l}\text { Major b } \\
\text { (ISTH) }\end{array}$ & $\begin{array}{l}\text { Defined as clinically overt bleeding that is associated with: } \\
\text { A fall in haemoglobin of } 2 \mathrm{~g} / \mathrm{L} \text { or more } \\
\text { A transfusion of } \geq 2 \text { units of packed red blood cells or whole blood } \\
\text { Bleeding in a critical site: intracranial, intraspinal, intraocular, pericardial, intra-articular, intramuscular with compartment } \\
\text { syndrome, retroperitoneal }\end{array}$ \\
\hline $\begin{array}{l}\text { Clinically relevant } \\
\text { nonmajor bleeding }\end{array}$ & $\begin{array}{l}\text { Defined as overt bleeding not meeting the criteria for major bleeding but associated with medical intervention, an } \\
\text { unscheduled contact (visit or telephone call) with a physician, (temporary) cessation of study treatment, or discomfort for the } \\
\text { patient, including: } \\
\text { Any bleeding compromising haemodynamics } \\
\text { Any bleeding leading to hospitalisation } \\
\text { Subcutaneous (skin) hematoma if the size is }>25 \mathrm{~cm} 2 \text {, or }>100 \mathrm{~cm} 2 \text { if provoked } \\
\text { Intramuscular haematoma } \\
\text { Epistaxis lasting for more than } 5 \mathrm{~min} \text {, if the episode was repetitive (ie, two or more episodes of true bleeding, that is, not } \\
\text { spots on a handkerchief, within } 24 \text { hours), or led to an intervention (packing, electrocoagulation etc) } \\
\text { Gingival bleeding if it occurred spontaneously (ie, unrelated to tooth brushing or eating), or if it lasted for more than } 5 \text { min } \\
\text { Haematuria if it was macroscopic, and either spontaneous or lasting for more than } 24 \text { hours after instrumentation (eg, } \\
\text { catheter placement or surgery) of the urogenital tract } \\
\text { Macroscopic gastrointestinal haemorrhage: at least one episode of melena/hematemesis, if clinically apparent and } \\
\text { haemoccult positive } \\
\text { Rectal blood loss, if more than a few spots on toilet paper } \\
\text { Haemoptysis, if more than a few speckles in the sputum and not occurring within the context of PE } \\
\text { Any other bleeding type that was considered to have clinical consequences for a patient }\end{array}$ \\
\hline
\end{tabular}

Minor bleeding Defined as other overt bleeding events that do not fulfil the criteria of a major bleeding event or a clinically relevant non-major bleeding event (eg, epistaxis that does not require medical attention).

Transient ischaemic Defined as new neurological symptoms or deficit lasting less than 24 hours without acute infarction on CT or MRI (if available) attack

Myocardial infarction

(fourth universal

definition)

*Detection of a rise and/or fall of cardiac Troponin values with at least one value above the 99th percentile URL and with at

least one of the following:

- Symptoms of acute myocardial ischemia

- New ischaemic ECG changes

- Development of pathological Q waves

- Imaging evidence of new loss of viable myocardium or new regional wall motion abnormality in a pattern consistent with an ischaemic aetiology

- Identification of a coronary thrombus by angiographic methods or autopsy. 


\section{Table 1 Continued}

Cause of death will be classified as follows:
Cardiovascular
Ischaemic stroke
Haemorrhagic stroke
Systemic or pulmonary embolism
Oner cardiovascular (ie, myocardial infarction, sudden death, heart failure) and unobserved deaths
Non-cardiovascular
Bleeding
Other non-cardiovascular (ie, malignancy, infection, trauma, pulmonary causes of death)
Unknown death: observed deaths of unknown cause

*Intracranial bleeding that met the definition of stroke was included in both stroke and major bleeding endpoints.

CTPA, CT pulmonary angiography/angiogram; ISTH, International Society on Thrombosis and Haemostasis; MI, myocardial infarction; PE, pulmonary embolism; ; VQ scan, ventilation/perfusion lung scintigraphy.

assessed by means of the Medical Outcomes Study 36-Item Short-Form General Health Survey questionnaire. ${ }^{17}$

\section{STUDY PLAN}

To facilitate a timely and efficient patient recruitment, PROTECT-AR will take advantage of preexisting local registries of ACHD patients, available to the study's centres. ${ }^{18}$ The study includes prescreening, screening and follow-up phases, executed in parallel (figure 1).

In the prescreening phase, available local registries will be scanned for subjects with a positive record regarding OAC use. To reduce selection bias, all of these subjects will be reached by a telephone call and briefly questioned on current apixaban use, core inclusion and exclusion criteria and on their ability and principal willingness to participate. Qualifying prescreened subjects will be asked to undergo a screening visit at one of the study's involved centres. Prescreening aside, active screening will also be performed to all consecutive patients who present for a routine exam, or are acutely hospitalised in a study's ACHD centre. All screened subjects will be documented in an anonymous $\log$ file, along with the reasons for screening failure (eg, ineligibility or refusal to provide consent).

At enrolment, baseline data on the clinical profile of all subjects who sign the written informed consent will be captured by local site staff (box 1). Emphasis will be placed on type of CHD (classified as outlined in the 2018 American Heart Association/American College of Cardiology guideline for the Management of ACHD),${ }^{19}$ prior antithrombotic medication, prior thromboembolic and bleeding events and associated risk scores. ${ }^{2021}$

Follow-up data regarding the occurrence of the trial's endpoints will be prospectively collected by telephone or in the ACHD centre should the patient return for a routine visit (or an acute hospitalisation), initially 1 month after enrollment and at 6-month intervals. Patients will be instructed to report to the study centre earlier if they
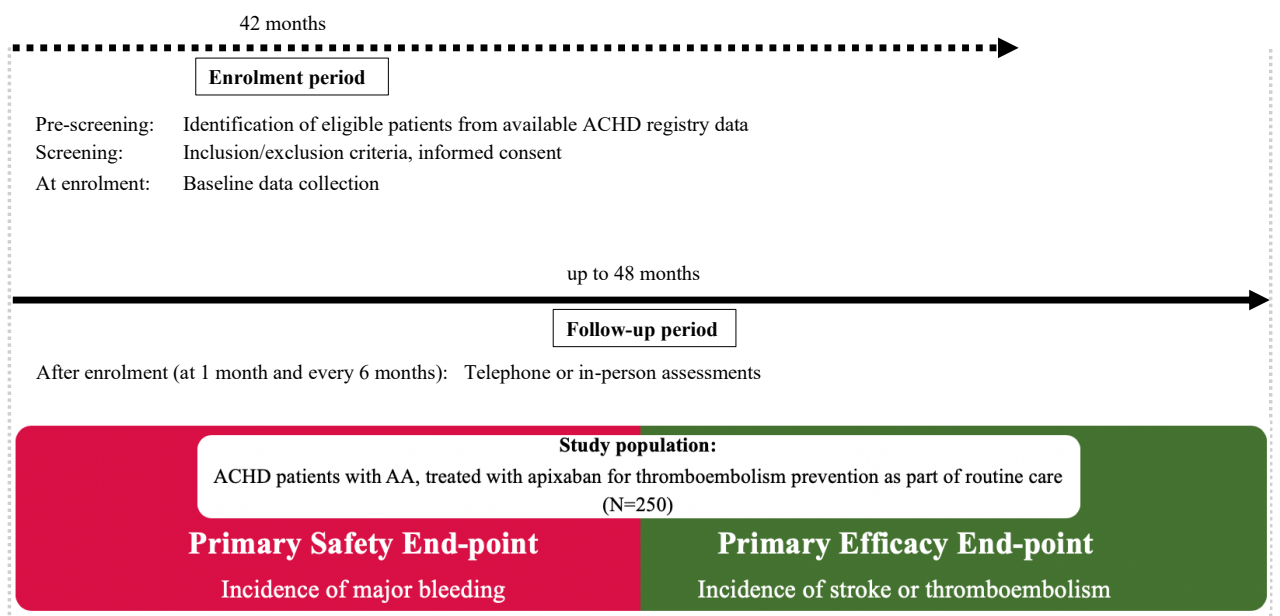

Study

Initiation Date

Study

Termination Date

(at $\sim 500$ patient-

years of follow-up)

Figure 1 Study plan of the PROTECT-AR study. AA, atrial arrhythmias; ACHD, adults with congenital heart disease; PROTECTAR, PReventiOn of ThromboEmbolism in Adults with Congenital HearT disease and Atrial aRrhythmias. 


\section{Box 1 Baseline data collection}

Sociodemographic profile (gender, age, weight, height, body mass index).

- Type of ACHD, stratified by:

- Anatomical complexity (I: simple, II: moderate, III: complex).

- Physiological stage (A, B, C, D).

- Date of AA diagnosis.

- Type of AA, confirmed by means of available 12-lead ECG and Holter-ECG, or electrophysiology study.

- Pattern of AA (first diagnosed, paroxysmal, persistent, permanent).

- History of interventions.

- Defect repair, including number of surgeries and presence of bioprosthetic valves.

- Cardioversion, ablation, pacemaker or intracardiac defibrillator implantation.

- Type of prior treatment:

- Antithrombotic therapy (VKA, NOAC, no OAC) including date of treatment initiation and patient-reported adherence.

- Rate control (b-blocker, calcium-channel blocker, digitalis).

- Rhythm control (propafenone, flecainide, sotalol, amiodarone).

- Other (nonsteroidal anti-inflammatory drugs, antidepressants).

- History of stroke, transient ischemicischaemic attack, systemic, pulmonary or venous thromboembolism, intracardiac thrombi, major bleeding or clinically relevant nonmajor bleeding

- Other comorbidities (hypertension, diabetes mellitus, dyslipidaemia, smoking, alcohol use, vascular disease, heart failure, thyroid disease, gastrointestinal disease, chronic kidney disease, chronic obstructive pulmonary disease, liver disease).

- Prior hospitalisation or healthcare resource utilisation due to AArelated complications.

- $\mathrm{CHA}_{2} \mathrm{DS}_{2}$-Vasc (Congestive heart failure, hypertension, age, diabetes mellitus, prior stroke or transient ischemic attack) and HAS-BLED (Hypertension, abnormal renal or liver function, stroke, bleeding, labile INR, elderly) score.

- Apixaban treatment decision (patient or physician).

- Other assessments, if performed as part of routine care (or most recent available):

- Vital signs (blood pressure, heart rate).

- Laboratory data (complete blood count, liver function tests, renal function tests, coagulation profile, $\mathrm{N}$-terminal pro-BNP [NT-pro-BNP]).

- Echocardiographic data (systemic ventricle ejection fraction, left atrium diameter, presence and severity of valvular disease).

- Symptom burden (modified European Heart Rhythm Association [mEHRA] symptom scale) and quality of life measures (SF-36 score).

$\mathrm{AA}$, atrial arrhythmias; $\mathrm{ACHD}$, adults with congenital heart disease; $\mathrm{CHA}_{2} \mathrm{DS}_{2}-$ Vasc, Congestive heart failure, hypertension, age, diabetes mellitus, prior stroke or transient ischemic attack ( $\mathrm{CHA}_{2} \mathrm{DS}_{2}$-Vasc); HAS-BLED, Hypertension, abnormal renal or liver function, stroke, bleeding, labile INR, elderly; mEHRA, modified European Heart Rhythm Association; NOAC, non-vitamin K oral anticoagulant; OAC, oral anticoagulant; VKA, vitamin-K antagonist; SF-36 to 36-Item Short-Form General Health Survey.

have symptoms suggestive of stroke, systemic, pulmonary embolism or major bleeding. The final follow-up visit will coincide with the date the study reaches the predetermined number of person-years of follow-up (ie, study termination date).

\section{DRUG ADMINISTRATION}

Given the study's noninterventional nature, decisions about apixaban prescription, including dose and duration of therapy, as well as any background therapy, laboratory or other investigations will be left to the discretion of the treating physician. Nevertheless, the study encourages management according to current guideline-based and label-based recommendations. In specific, the European Society of Cardiology guidelines suggest OAC in all patients with ACHD with AA and intracardiac repair, cyanosis, Fontan palliation or systemic right ventricle. In less complex forms of ACHD, OAC is suggested if $\mathrm{CHA}_{2} \mathrm{DS}_{2}$-Vasc (congestive heart failure, hypertension, age $\geq 75$ years, diabetes mellitus, stroke/transient ischaemic attack, vascular disease, age $65-74$ years, sex category) score is $\geq 1$ (class of recommendation IIa, level of evidence C). ${ }^{4}$ The recommended dose of apixaban is $2.5 \mathrm{mg}$ two times a day if patients meet two or more of the following criteria: an age of at least 80 years, a body weight of no more than $60 \mathrm{~kg}$, or a serum creatinine level of $1.5 \mathrm{mg} / \mathrm{dL}$ or more. Apixaban $2.5 \mathrm{mg}$ two times a day is also approved for treatment of patients with severe renal impairment (creatinine clearance $15-30 \mathrm{~mL} / \mathrm{min}$ ), but not end-stage renal impairment (creatinine clearance $<15 \mathrm{~mL} / \mathrm{min}$ ) or dialysis. The drug is also contraindicated in severe hepatic impairment (Child-Pugh class C). For all other cases of non-valvular AA, apixaban $5 \mathrm{mg}$ two times a day is recommended. ${ }^{22}$

\section{STATISTICAL CONSIDERATIONS}

\section{Sample size}

The sample size of PROTECT-AR was calculated by testing a hypothesis of non-inferiority between apixaban and warfarin, with respect to the study's primary efficacy endpoint (ie, stroke or thromboembolism). Designing an adequately powered randomised, controlled trial was prohibitive in terms of time, cost and sample size. Hence, we implemented a single-arm, historical control design. Historical cohort data suggest an annual thromboembolism rate of $1.2 \%$ in patients with ACHD with AA and no mechanical valves, under warfarin treatment. ${ }^{23}$ In ARISTOTLE, the HR for stroke or systemic embolism when comparing apixaban with warfarin was $0.79 .{ }^{6}$ Accordingly, we would expect the annual rate of the primary efficacy endpoint in our population on apixaban to be $0.95 \%$. On the basis of multiple scenarios balancing practicality and clinical meaningfulness, the non-inferiority hypothesis was accepted should the actual annual rate of the primary efficacy endpoint not exceed the rate in the historical cohort by more than $1.8 \%$ in absolute terms (ie, if it is not higher than $3 \%$ ). This required 478 patient-years to provide power of $90 \%$ at a one-sided alpha level of 0.025 . These patient-years were also considered acceptable to determine non-inferiority with respect to the study's primary safety endpoint, yielding power of $84 \%$ at a onesided alpha level of 0.02 to detect a non-inferiority rate of $2.5 \%$, when the projected actual annual major bleeding 
rate is $0.76 \%{ }^{6}{ }^{23}$ We anticipate a minimal attrition rate given the close follow-up of patients in the ACHD sites, and thus, aim to recruit a total of 250 patients with a mean follow-up of 2 years, to achieve approximately 500 patient-years of exposure to apixaban.

\section{STATISTICAL ANALYSIS}

The safety and efficacy assessment will be based on calculations of the incidence rate of adjudicated clinical events, with baseline being the time of enrolment. Only follow-up and events occurring prospectively, after enrolment, will be counted in the analyses. Follow-up data will be censored on the earliest of the following: the date of death (if applicable), and the study termination date (ie, the date when the prespecified patient-years will be reached). Incidence proportions (patients with events/ number of treated patients) and incidence rates (events per 100 patient-years) of all binary primary and secondary endpoints will be calculated, with corresponding $95 \%$ CIs. Time-to-event endpoints will be plotted with the use of the Kaplan-Meier method, measured from the time of enrollment to the time of occurrence of the first event. Differences in event rates between new and experienced apixaban users, patients of different disease complexity or AA type will be assessed by using the log-rank test or Cox proportional regression HR. Data will be primarily analysed according to the intention-to-treat principle, that is, without reference to treatment compliance or changes. In a secondary on-treatment analysis, patients will be censored in case of apixaban discontinuation or switching to a different OAC. Descriptive statistics will be used to summarise baseline characteristics. Continuous variables will be expressed as mean $\pm \mathrm{SD}$ or median and interquartile range ((IQR); 25th, 75th percentile) depending on normality of the distribution. Categorical values will be presented as frequencies and percentages. Intervariable comparisons will be conducted, using analysis of variance, $\chi^{2}$ test or Fisher's exact tests where appropriate. Data management and statistical analyses will be performed using Stata software, version 16 (StataCorp).

\section{Ethics and dissemination}

The study will be conducted in keeping with International Conference on Harmonisation Good Clinical Practice guidelines, the Declaration of Helsinki, and applicable laws and regulations. ${ }^{24}$ All participating sites have obtained approval from appropriate independent ethics committees or institutional review boards, and patients will provide written informed consent before study entry. Results will be published as peer-reviewed articles in international journals and communicated through national and international conferences.

\section{Subject confidentiality}

Data will be centrally stored in a structured electronic database and will be only accessible by study staff. Strict subject confidentiality will be maintained through subject identification codes.

\section{Data and safety monitoring}

At multiple time points a data and safety monitoring board consisting of study investigators and an independent statistician will review accumulating data for quality and safety and will report back to the steering committee of the study. Serious adverse events will be reported by the steering committee to Pfizer within 24 hours of first awareness of the event.

\section{Patient and public involvement}

There has been no public or patient involvement in the design of this study. The study results will be disseminated to the participating patients via the investigators, once they become published in a peer-reviewed journal.

\section{DISCUSSION}

In this study, we aim to test the hypothesis that routine, 'real-world' use of apixaban in patients with ACHD and non-valvular AA, is associated with a non-inferior risk of stroke or thromboembolic events and a non-inferior risk of major bleeding events compared with historical cohort data on warfarin. The lack of documented pragmatic experience with NOACs in ACHD is reflected in the guidelines: The 2018 AHA/ACC guidelines for the management of ACHD strongly encourage future research to identify patients with ACHD who can use NOACs instead of warfarin. ${ }^{25}$ The 2014 PACES/HRS expert consensus statement on the management of arrhythmias in ACHD issued weak recommendations for NOACs in ACHD, pending safety and efficacy data. ${ }^{26}$ So far there have been only two prospective studies assessing the safety and efficacy of NOAC treatment in the ACHD setting, ${ }^{710}$ while most available data stem from retrospective studies. ${ }^{27-29}$ These data are limited by heterogeneity regarding OAC treatment, since patients were under a mixture of warfarin or NOAC agents. ${ }^{30}$ PROTECT-AR's non-interventional, longitudinal design is meant to fill this unmet clinical need, particularly for apixaban. Focusing research on apixaban in 'real-world' ACHD patients is backed up by scientific merit; ARISTOTLE and ENGAGE TIMI 48 were the only landmark randomised controlled NOAC trials to include patients with bioprosthetic valves or valve repair, ${ }^{31}$ which are common features in ACHD. Furthermore, complex CHD, especially Fontan, is associated with varying degrees of hepatic insufficiency. In these cases, VKA use is particularly challenging due to intrinsically elevated INR and an excess difficulty in dose adjustment. ${ }^{32}$ Apixaban is one of the NOACs allowed in mild to moderate hepatic impairment (Child-Pugh class A or B), whereas its metabolism is predominantly hepatic via CYP3A4/5. ${ }^{22}$ The limited clinical experience mandates further prospective studies, in the adult and paediatric setting alike; two trials on apixaban and rivaroxaban in paediatric patients with CHD are expected to 
shed light on this unexplored territory. ${ }^{33} 34$ The independent endpoint adjudication of PROTECT-AR will allow for comprehensive, quality details on the endpoints of interest, in compliance with predefined standards. In a similar fashion to the NOTE (NOACs for thromboembolic prevention in patients with CHD) study ${ }^{10}$ PROTECT-AR exploits an existing pool of phenotypically characterised patients with ACHD, nested into active, local ACHD-allcomers registries, from which ongoing apixaban users are to be selected. Such use of already available resources is expected to offer several advantages compared with other studies: First, it will reduce time and recruitment costs. Second, it will reduce selection bias on the site level since some patients will be enrolled at the study's sites although being followed at other sites contributing to the registries. Thus, although limited to a single country, our cohort will, to some extent, reflect a diverse ACHD population on apixaban that is representative of a nationwide distribution of care settings.

\section{LIMITATIONS}

Despite its statistical and theoretical plausibility, PROTECT-AR shares the same limitation with previous NOAC studies in the context of ACHD, of not being a formal non-inferiority trial of apixaban vs warfarin. Such a trial was deemed not to be feasible given the need to include an actual warfarin control group and to enrol at least 18000 patients in total to establish non-inferiority according to industry standards. ${ }^{635}$ Consequently, the present study should be seen as an exploratory safety and efficacy evaluation confined to apixaban in patients with ACHD with AA. Being a real-world study, PROTECT-AR is subject to various forms of selection bias. The pooling of OAC-experienced patients from local registries might lead to underestimation of adverse events, since these tend to occur during the critical period of OAC initiation. ${ }^{36}$ The study's single-arm, open-label design can introduce bias related to knowledge about treatment. It is likely that a clinician will be selecting patients most likely to benefit, and without risk of complication, from apixaban. Knowledge about the study in the involved ACHD sites could incline physicians to switch from other OACs or initiate apixaban more often, in order to aid recruitment. In addition, patients agreeing to participate in the study may, to some extent, be prone to self-selection, for reasons such as high motivation or perceived high risk of stroke or bleeding.

\footnotetext{
Author affiliations

${ }^{1}$ Cardiology, University General Hospital of Thessaloniki AHEPA, Thessaloniki, Greece ${ }^{2}$ Cardiology, Onassis Cardiac Surgery Centre, Athens, Attica, Greece

${ }^{3}$ Cardiology, General University Hospital Attikon, Athens, Attica, Greece

${ }^{4}$ Paediatric Cardiology \& Adult Congenital Heart Disease, Mitera, Hygeia Group, Athens, Attica, Greece

${ }^{5}$ Division of Biomedical Engineering and Imaging Sciences, King's College, London, England
}

Acknowledgements We thank all the patients, investigators, and site staff for participating in this study.
Contributors AKa wrote funding application and study protocol, designed data collection CRF, drafted manuscript. ID and GR wrote funding application, critiqued funding application and manuscript, wrote ethics application. DN, AKo, DK, SA, AF, AT, DA, ON, SL, TM, SAM and HK critiqued funding application and manuscript. GG conceived study, critiqued funding application and study protocol, critiqued manuscript.

Funding PROTECT-AR is an investigator-initiated study, supported by Pfizer through European Thrombosis Investigator Initiated Research Program (ERISTA, Reference Number: WI246683).

Disclaimer Pfizer had no role in the design or conduct of the study; preparation, review or approval of the manuscript; and decision to submit the manuscript for publication.

Competing interests GG and HK have received grants and/or personal fees from Pfizer.

Patient and public involvement Patients and/or the public were not involved in the design, or conduct, or reporting, or dissemination plans of this research.

Patient consent for publication Not required.

Ethics approval The study has been approved by the (1) Institutional review board and ethics and deontology committee of AHEPA University Hospital, Thessaloniki, Greece: 145/ 05.04.2019. (2) Institutional review board of Attikon University Hospital, Athens, Greece: 296 28.05.2019. (3) Institutional review board and ethics and deontology committee of Onassis Cardiac Surgery Center, Athens, Greece: 642/21.03.2019. (4) Institutional review board of Mitera Childrens' Hospital, Athens, Greece: 05.03.2019.

Provenance and peer review Not commissioned; externally peer reviewed.

Open access This is an open access article distributed in accordance with the Creative Commons Attribution Non Commercial (CC BY-NC 4.0) license, which permits others to distribute, remix, adapt, build upon this work non-commercially, and license their derivative works on different terms, provided the original work is properly cited, appropriate credit is given, any changes made indicated, and the use is non-commercial. See: http://creativecommons.org/licenses/by-nc/4.0/.

\section{ORCID iD}

Anastasios Kartas http://orcid.org/0000-0002-1170-9133

\section{REFERENCES}

1 Hoffmann A, Chockalingam P, Balint $\mathrm{OH}$, et al. Cerebrovascular accidents in adult patients with congenital heart disease. Heart 2010;96:1223-6.

2 Bouchardy J, Therrien J, Pilote L, et al. Atrial arrhythmias in adults with congenital heart disease. Circulation 2009;120:1679-86.

3 Lasa JJ, Glatz AC, Daga A, et al. Prevalence of arrhythmias late after the Fontan operation. Am J Cardiol 2014;113:1184-8.

4 Kirchhof P, Benussi S, Kotecha D, et al. 2016 ESC guidelines for the management of atrial fibrillation developed in collaboration with EACTS. Eur Heart J 2016;37:2893-962.

5 Giannakoulas G, Boutsikou M. The Gordian knot of thromboembolism in congenital heart disease. Heart 2015;101:1523-4.

6 Granger CB, Alexander JH, McMurray JJV, et al. Apixaban versus warfarin in patients with atrial fibrillation. $N$ Engl J Med 2011;365:981-92.

7 Pujol C, Niesert A-C, Engelhardt A, et al. Usefulness of direct oral anticoagulants in adult congenital heart disease. Am J Cardiol 2016;117:450-5.

8 Yang H, Bouma BJ, Mulder BJM, et al. Is initiating NOACs for atrial arrhythmias safe in adults with congenital heart disease? Cardiovasc Drugs Ther 2017;31:413-7.

9 Arslani K, Notz L, Zurek M, et al. Anticoagulation practices in adults with congenital heart disease and atrial arrhythmias in Switzerland. Congenit Heart Dis 2018;13:678-84.

10 Yang H, Bouma BJ, Dimopoulos K, et al. Non-Vitamin K antagonist oral anticoagulants (NOACs) for thromboembolic prevention, are they safe in congenital heart disease? results of a worldwide study. Int $J$ Cardiol 2020;299:123-30.

11 Hicks KA, Mahaffey KW, Mehran R, et al. 2017 cardiovascular and stroke endpoint definitions for clinical trials. Circulation 2018;137:961-72.

12 Schulman S, Angerås U, Bergqvist D, et al. Definition of major bleeding in clinical investigations of antihemostatic medicinal products in surgical patients. J Thromb Haemost 2010;8:202-4. 
13 Kaatz S, Ahmad D, Spyropoulos AC, et al. Definition of clinically relevant non-major bleeding in studies of anticoagulants in atrial fibrillation and venous thromboembolic disease in non-surgical patients: communication from the SSC of the ISTH. J Thromb Haemost : 2015;13:2119-26.

14 Weitz JI, Lensing AWA, Prins MH, et al. Rivaroxaban or aspirin for extended treatment of venous thromboembolism. $N$ Engl J Med Overseas Ed 2017;376:1211-22.

15 Agnelli G, Buller HR, Cohen A, et al. Apixaban for extended treatment of venous thromboembolism. N Engl J Med 2013;368:699-708.

16 Ageno W, Mantovani LG, Haas S, et al. XALIA: rationale and design of a non-interventional study of rivaroxaban compared with standard therapy for initial and long-term anticoagulation in deep vein thrombosis. Thromb J 2014;12:16.

17 Ware JE. Sf-36 health survey update. Spine 2000;25:3130-9.

18 Giannakoulas G, Vasiliadis K, Frogoudaki A, et al. Adult congenital heart disease in Greece: preliminary data from the challenge registry. Int J Cardiol 2017;245:109-13.

19 Stout KK, Daniels CJ, Aboulhosn JA, et al. 2018 AHAVACC Guideline for the Management of Adults With Congenital Heart Disease: Executive Summary: A Report of the American College of Cardiology/American Heart Association Task Force on Clinical Practice Guidelines. J Am Coll Cardiol 2019;73:1494-563.

20 Lip GYH, Nieuwlaat R, Pisters R, et al. Refining clinical risk stratification for predicting stroke and thromboembolism in atrial fibrillation using a novel risk factor-based approach: the Euro heart survey on atrial fibrillation. Chest 2010;137:263-72.

21 Pisters R, Lane DA, Nieuwlaat R, et al. A novel user-friendly score (HAS-BLED) to assess 1-year risk of major bleeding in patients with atrial fibrillation: the Euro heart survey. Chest 2010;138:1093-100.

22 Steffel J, Verhamme P, Potpara TS, et al. The 2018 European heart rhythm association practical guide on the use of non-vitamin $\mathrm{K}$ antagonist oral anticoagulants in patients with atrial fibrillation. Eur Heart J 2018:1-64.

23 Heidendael JF, Bokma JP, de Groot JR, et al. Weighing the risks: thrombotic and bleeding events in adults with atrial arrhythmias and congenital heart disease. Int J Cardiol 2015;186:315-20.

24 World Medical Association. World Medical association Declaration of Helsinki: ethical principles for medical research involving human subjects. JAMA 2013;310:2191-4.
25 Aboulhosn JA, Fuller S, Bozkurt B, et al. 2018 AHA/ACC guideline for the management of adults with congenital heart disease ACC/AHA Task force members. J Am Coll Cardiol 2018.

26 Khairy P, Warnes CA, Van Hare GF, et al. PACES/HRS expert consensus statement on the recognition and management of arrhythmias in adult congenital heart disease: developed in partnership between the pediatric and congenital electrophysiology Society (PACES) and the heart rhythm Society (HRS). En Hear Rhythm 2014;11:e102-65.

27 Khairy P, Aboulhosn J, Broberg CS, et al. Thromboprophylaxis for atrial arrhythmias in congenital heart disease: a multicenter study. Int J Cardiol 2016;223:729-35.

28 Georgekutty J, Kazerouninia A, Wang Y, et al. Novel oral anticoagulant use in adult Fontan patients: a single center experience. Congenit Heart Dis 2018;13:541-7.

29 Heidendael JF, Bokma JP, de Groot JR, et al. Weighing the risks: thrombotic and bleeding events in adults with atrial arrhythmias and congenital heart disease. Int J Cardiol 2015;186:315-20.

30 Stalikas N, Doundoulakis I, Karagiannidis E, et al. Non-Vitamin K ora anticoagulants in adults with congenital heart disease: a systematic review. J Clin Med 2020;9. doi:10.3390/jcm9061794. [Epub ahead of print: 09 Jun 2020].

31 De Caterina R, Camm AJ. What is 'valvular' atrial fibrillation? A reappraisal. Eur Heart J 2014;35:3328-35.

32 Efird LM, Mishkin DS, Berlowitz DR, et al. Stratifying the risks of oral anticoagulation in patients with liver disease. Circ Cardiovasc Qual Outcomes 2014;7:461-7.

33 Payne RM, Burns KM, Glatz AC, et al. A multi-national trial of a direct oral anticoagulant in children with cardiac disease: design and rationale of the safety of apixaban on pediatric heart disease on the preventioN of embolism (saxophone) study. Am Heart $J$ 2019;217:52-63.

34 Pina LM, Dong X, Zhang L, et al. Rivaroxaban, a direct factor Xa inhibitor, versus acetylsalicylic acid as thromboprophylaxis in children post-Fontan procedure: rationale and design of a prospective, randomized trial (the universe study). Am Heart J 2019;213:97-104.

35 U.S. Department of health and human services food and drug administration. Non-Inferiority Clinical Trials to Establish Effectiveness: Guidance for Industry, 2016.

36 Bassand J-P, Accetta G, Camm AJ, et al. Two-Year outcomes of patients with newly diagnosed atrial fibrillation: results from GARFIELD-AF. Eur Heart J 2016;37:2882-9. 